\title{
Topoisomerase $2 \alpha$ and thymidylate synthase expression in adrenocortical cancer
}

\author{
Elisa Roca', Alfredo Berruti1, Silviu Sbiera2, Ida Rapa3, Ester Oneda', Paola Sperone4, \\ Cristina L Ronchi², Laura Ferrari', Salvatore Grisanti', Antonina Germano5, \\ Barbara Zaggia5, Giorgio Vittorio Scagliotti4, Martin Fassnacht ${ }^{2}$, Marco Volante ${ }^{3}$, \\ Massimo Terzolo 5 and Mauro Papotti6
}

1Department of Medical and Surgical Specialties, Radiological Sciences, and Public Health, University of Brescia, Oncology Unit, ASST Spedali Civili di Brescia, Brescia, Italy

2Division of Endocrinology and Diabetes, Department of Internal Medicine I, University Hospital, University of Wuerzburg, Wuerzburg, Germany

${ }^{3}$ Department of Oncology, University of Turin, Pathology Unit, San Luigi Gonzaga Hospital, Orbassano, Italy ${ }^{4}$ Department of Oncology, University of Turin, Medical Oncology, San Luigi Gonzaga Hospital, Orbassano, Italy 5Department of Clinical and Biological Sciences, University of Turin, Internal Medicine 1, San Luigi Gonzaga Hospital, Orbassano, Italy

${ }^{6}$ Department of Oncology, University of Turin, Pathology Unit, City of Health and Science Hospital, Turin, Italy

Correspondence should be addressed to A Berruti Email alfredo.berruti@unibs.it

\begin{abstract}
Topoisomerase II alpha (TOP2A) and thymidylate synthase (TS) are known prognostic parameters in several tumors and also predictors of efficacy of anthracyclines, topoisomerase inhibitors and fluoropirimidines, respectively. Expression of TOP2A and TS mRNA was assessed in 98 patients with adrenocortical carcinoma (ACC) and protein expression was assessed by immunohistochemistry in a subset of 39 tumors. Ninety-two patients were radically resected for stage II-III disease and 38 of them received adjuvant mitotane. Twenty-six patients with metastatic disease received the EDP-M (etoposide, doxorubicin, Adriamycin, cisplatin plus mitotane). TOP2A and TS expression in ACC tissue was directly correlated with the clinical data. Both markers were not associated with either disease free survival (DFS) or overall survival (OS) in multivariate analyses and failed to be associated to mitotane efficacy. Disease response or stabilization to EDP-M treatment was observed in 12/17 (71\%) and 1/9 (11\%) patients with high and low TOP2A expressing tumors $(P=0.0039)$ and $9 / 13(69 \%)$ and $4 / 13(31 \%)$ patients with high and low TS expressing ACC, respectively $(P=0.049)$. High TOP2A expression was significantly associated with longer time to progression (TTP) after EDP-M. TOP2A and TS proteins assessed by immunohistochemistry significantly correlated with mRNA expression. Immunohistochemical TOP2A expression was associated with a non-significant better response and longer TTP after EDP-M. TOP2A and TS were neither prognostic nor predictive of mitotane efficacy in ACC patients. The predictive role of TOP2A expression of EDP-M activity suggests a significant contribution of Adriamycin and etoposide for the efficacy of the EDP scheme.
\end{abstract}

\section{Key Words}

- adrenocortical carcinoma

- topoisomerase alpha 2

- thymidylate synthase

- prognostic and predictive factors 


\section{Introduction}

Adrenocortical carcinoma (ACC) is a rare and aggressive malignant tumor (Fassnacht et al. 2011, Terzolo et al. 2013). Surgery is the mainstay of therapy. Although complete surgical removal of ACC is the only potentially curative approach, most of the radically resected patients are destined to relapse, often with metastases (Bellantone et al. 1997, Icard et al. 2001). Mitotane is the reference systemic therapy for ACC (Hahner \& Fassnacht 2005). On the basis of the results of a large retrospective multicentric study carried out at several referral centers in Italy and Germany (Terzolo et al. 2007), the drug is recommended to be administered in adjuvant setting in radically resected ACC with high risk of recurrence (Berruti et al. 2010, 2012). The recent finding that mitotane serum levels are prognostic in patients receiving the drug as adjuvant therapy provides further evidence in favor of the efficacy of this drug in this setting (Terzolo et al. 2013). However, few prognostic factors are currently available to identify the patient risk of relapse and death of disease. Currently only disease stage, completeness of initial resection, and proliferation index are widely accepted prognostic factors (Volante et al. 2008, Berruti et al. 2010, Beuschlein et al 2015). In the patients with metastatic disease at diagnosis or showing distant recurrence after surgery, chemotherapy plus mitotane is the best treatment strategy. The results of a multinational prospective randomized clinical study have established the combination chemotherapy with the topoisomerase II inhibitor etoposide, anthracycline, doxorubicin, cisplatin plus mitotane (EDP-M) as the reference regimen for this rare disease (Fassnacht et al. 2012). Adjuvant mitotane therapy and EDP-M however, are toxic and their overall efficacies limited. Therefore, the establishment of predictive factors that identify a subset of patients, in whom a certain treatment would be more effective, would be highly desirable.

We have previously evaluated the expression of ribonucleotide reductase large subunit 1 (RRM1) and ERCC1 genes in a multicenter (German and Italian) cohort of ACC patients and provided the first evidence in vitro and in vivo that RRM1 gene expression levels are functionally associated to mitotane sensitivity and predict response to mitotane treatment in adjuvant setting (Volante et al. 2012).

In the same series we now assessed the expression of two other biomarkers with potential prognostic impact: the topoisomerase II alpha (TOP2A), an enzyme responsible for transcription, replication and chromosome condensation and segregation during cell division
(Kellner et al. 2002); and the thymidylate synthase (TS), an enzyme involved in nucleotide metabolism (Lv et al. 2013). TOP2A is also a well-known predictor of efficacy of anthracyclines and topoisomerase inhibitors (Wang et al. 2012) and TS is predictive of the efficacy of fluoropyrimidines (Formentini et al. 2004). In the present study, we explored the prognostic significance of these markers in ACC as well as their predictive role for efficacy of adjuvant mitotane and of EDP-M in patients with advanced disease.

\section{Materials and methods}

\section{Patients}

Ninety-eight patients with radically resected ACC between 1989 and 2007 at the San Luigi Hospital of Orbassano, University of Turin, Italy (51 patients) and 35 centers in Germany coordinated by the German ACC Registry (47 patients) were included according to the following eligibility criteria: (1) age of 18 years or older; (2) histologically confirmed diagnosis of ACC after central pathologic revision (MV, MP); (3) complete resection of primary ACC; and (4) availability of follow-up information, (5) availability of representative paraffin-embedded tissue block(s). All patients fulfilling these criteria were included in the study. Ninety-two patients were already considered in the previously published series that have tested ERCC1 and RRM1 (Volante et al. 2012). In the present paper we also considered six patients from the Italian archive that were excluded in the published series because they were metastatic at diagnosis. These patients were radically resected on primary tumor but not on metastatic disease. The diagnosis of ACC was based on the pathological Weiss score (Weiss et al. 1989). Variables recorded included age, sex, hormone secretion, ENSAT stage at diagnosis (Fassnacht et al. 2009), initial therapeutic options including primary surgery, disease-free survival (DFS) for patients initially radically resected, defined by the time elapsing from diagnosis to either disease relapse or patient death, overall survival (OS), calculated from diagnosis till death, Weiss score, mitotic count, sites of metastases at the time of progression. ACC relapse was defined as the appearance of local recurrence or metastatic disease at imaging techniques during follow-up. Adjuvant mitotane was offered to patients considered at high risk of relapse, in presence of the following criteria: (1) stage III ACC; (2) high mitotic index. When a post-operative adjunctive

Published by Bioscientifica Ltd 
measure was deemed necessary, a monitored mitotane treatment aiming at plasma concentrations between 14 and $20 \mathrm{mg} / \mathrm{L}$ (Baudin et al. 2001, Ceppi et al. 2008a,b) was employed. In the absence of intolerability to mitotane, treatment was scheduled for at least 2 years, or till ACC recurrence. Follow-up protocols were similar among the different centers including imaging (CT or MRI) of both chest and abdomen at baseline and thereafter every 3-6 months until disease progression or end of the study period. At each visit, the patients underwent physical examination, routine laboratory evaluation and hormonal work-up. Monitoring of mitotane concentrations was done in treated patients. For recurrent disease, radical surgery was performed if complete resection seemed feasible. In case of not resectable disease, patients received mitotane alone or chemotherapy plus mitotane according to disease aggressiveness, tumor bulk and previous mitotane exposure. Twenty-six patients, 4 metastatic at diagnosis and 22 with disease recurrence after primary surgery, received the combination of EDP-M. Patients gave informed consent for collecting tissue and clinical data and the study was approved by the ethics committees of both centers.

\section{RNA isolation from paraffin embedded tissues and quantitative real time PCR}

Representative tumor areas were dissected under stereomicroscopic assistance from $10 \mu \mathrm{m}$ sections of paraffin-embedded tissue in RNAse-free conditions. RNA isolation was performed by commercially available paraffin material RNA extraction kits according to manufacturer's instructions (High Pure RNA Paraffin Kit; Roche Applied Science). Complementary DNA was transcribed using $500 \mu \mathrm{g} / \mathrm{mL}$ oligo dT (Roche Applied Science) and $500 \mathrm{M}-\mathrm{MLV}$ RT $(200 \mathrm{U} / \mu \mathrm{L})$ (Invitrogen) according to standard protocols. Relative cDNA quantification of TS and TOP2A and of internal reference gene (beta-actin) was done in duplicate using a fluorescence-based real-time detection method (ABI PRISM 7900 Sequence Detection System-Taqman; Applied Biosystems, Life Technologies). Primers and probes sequences for TS and TOP2A and cycling conditions have already been published elsewhere (Ceppi et al. 2008a,b). The relative gene expression levels were expressed as ratios (differences between the $C_{\mathrm{t}}$ values) between 2 absolute measurements (genes of interest/internal reference gene).

Then, the $\Delta \Delta C_{\mathrm{t}}$ values were calculated subtracting $\Delta C_{\mathrm{t}}$ values of each case to the value of the case with the lowest expression, and converting the ratio by the $2^{-\Delta \Delta C_{t}}$ formula; cases were considered of low or high expression according to the median expression level obtained. A mixture containing Human Total RNA (Stratagene) was used as control calibrator on each plate.

\section{Immunohistochemical evaluation}

Five micrometer thick paraffin sections serial to those used for conventional hematoxylin and eosin staining were obtained for immunohistochemical reactions. The following antibodies were employed: TS (1/100, rabbit monoclonal, clone ERP4545) and TOP2A (diluted 1/100, rabbit monoclonal, clone EP1102y) (Abcam). Immunohistochemistry was performed in automated Ventana BenchMark Ultra instrument (Ventana, Roche). Immunoreactivity was scored using the $H$-score which is generated from the following equation: $H$-score $=\Sigma \mathrm{Pi}$ $(i+1)$, where ' $\Sigma$ ' represents the intensity of staining $(0-3+)$, and 'Pi' stands for the percentage of stained tumor cells (0-100\%).

\section{Statistical analyses}

Correlations between the expressions of TOP2A and TS genes, as well as between protein and mRNA expression were tested using the Spearman coefficient. Differences of

Table 1 Patients characteristics.

\begin{tabular}{lcc}
\hline Characteristics & $\boldsymbol{N}(\%)$ \\
\hline No. & 98 \\
Age (years) & \\
$\quad$ Median (range) & & \\
Sex, no. (\%) & $40(40.8)$ \\
$\quad$ Male & $58(59.2)$ \\
Female & \\
ENSAT ACC stage & $11(11.2)$ \\
I & $49(50.0)$ \\
II & $32(32.7)$ \\
III & $6(6.1)$ \\
IV & \\
Secreting tumor, no. (\%) & $51(52.0)$ \\
$\quad$ Non-secreting tumor & $30(30.6)$ \\
Cortisol \pm androgens & $8(8.2)$ \\
Androgens & $3(3.1)$ \\
Estradiol & $4(4.1)$ \\
Mineralcorticoids & $2(2.0)$ \\
$\quad$ Not available & \\
Weiss score & $5(3-9)$ \\
Median (range) & \\
Adjuvant mitotane, no. (\%) & $38(38.8)$ \\
Yes & $54(55.1)$ \\
No & $64(65.3)$ \\
Patients with recurring ACC, no. (\%) & $51(52.0)$ \\
Patients who died of ACC progression, no. (\%) & \\
\hline & \\
\hline
\end{tabular}

Published by Bioscientifica Ltd 
Table 2 Relationship of TOP2A and TS gene expression levels with either patient or tumor characteristics.

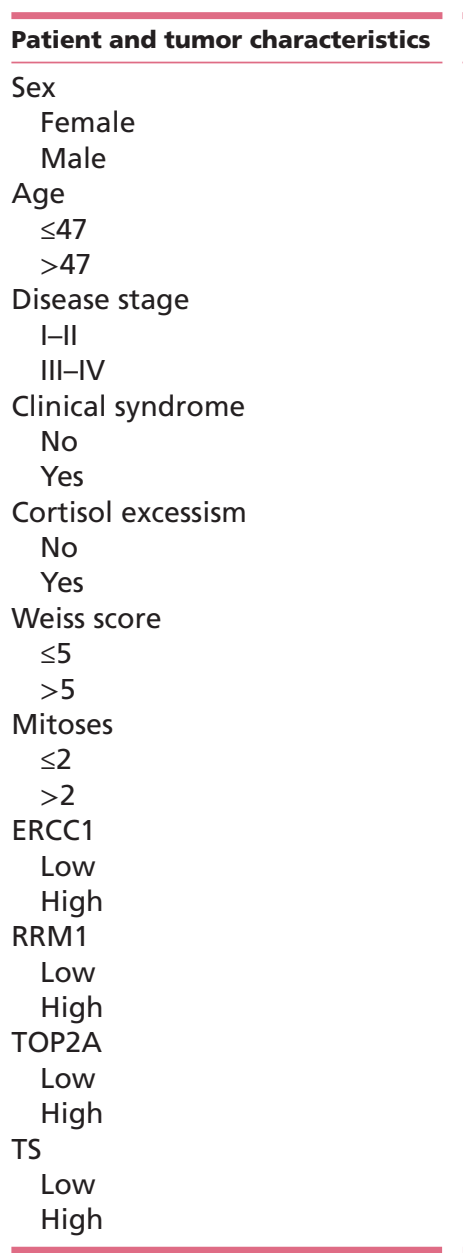

\begin{tabular}{|c|c|c|c|c|c|}
\hline TOP2A low & TOP2A high & $P$-value & TS low & TS high & $P$-value \\
\hline $33(67.35 \%)$ & $25(51.02 \%)$ & 0.10 & $30(62.50 \%)$ & $27(55.10 \%)$ & 0.46 \\
\hline $16(32.65 \%)$ & $24(48.98 \%)$ & & $18(37.50 \%)$ & $22(44.90 \%)$ & \\
\hline $25(51.02 \%)$ & $25(51.02 \%)$ & 1 & $26(54.17 \%)$ & $24(48.98 \%)$ & 0.61 \\
\hline $24(48.98 \%)$ & $24(48.98 \%)$ & & $22(45.83 \%)$ & $25(51.02 \%)$ & \\
\hline $32(65.31 \%)$ & $28(57.14 \%)$ & 0.41 & $32(66.67 \%)$ & $27(55.10 \%)$ & 0.24 \\
\hline $17(34.69 \%)$ & $21(42.86 \%)$ & & $16(33.33 \%)$ & $22(44.90 \%)$ & \\
\hline $28(57.14 \%)$ & $25(51.02 \%)$ & 0.54 & $25(52.08 \%)$ & $27(55.10 \%)$ & 0.76 \\
\hline $21(42.86 \%)$ & $24(48.98 \%)$ & & $23(47.92 \%)$ & $22(44.90 \%)$ & \\
\hline $34(69.39 \%)$ & $34(69.39 \%)$ & 1 & $33(68.75 \%)$ & $34(69.39 \%)$ & 0.94 \\
\hline $15(30.61 \%)$ & $15(30.61 \%)$ & & $15(31.25 \%)$ & $15(30.61 \%)$ & \\
\hline $27(56.25 \%)$ & $20(43.48 \%)$ & 0.21 & $28(60.87 \%)$ & $18(38.30 \%)$ & 0.03 \\
\hline $21(43.75 \%)$ & $26(56.52 \%)$ & & $18(39.13 \%)$ & $29(61.70 \%)$ & \\
\hline $30 / 51(62.5 \%)$ & $21 / 51(46.7 \%)$ & 0.12 & & & \\
\hline $18 / 42(37.5 \%)$ & $24 / 42(53.3 \%)$ & & & & \\
\hline $27(55.10 \%)$ & $24(48.98 \%)$ & 0.54 & $24(50.00 \%)$ & $27(55.10 \%)$ & 0.61 \\
\hline $22(44.90 \%)$ & $25(51.02 \%)$ & & $24(50.00 \%)$ & $22(44.90 \%)$ & \\
\hline $30(61.22 \%)$ & $19(38.78 \%)$ & 0.026 & $18(37.50 \%)$ & $31(63.27 \%)$ & 0.011 \\
\hline \multirow[t]{3}{*}{$19(38.78 \%)$} & $30(61.22 \%)$ & & $30(62.50 \%)$ & $18(36.73 \%)$ & \\
\hline & & & $31(64.58 \%)$ & $18(36.73 \%)$ & 0.006 \\
\hline & & & $17(35.42 \%)$ & $31(63.27 \%)$ & \\
\hline $31(63.27 \%)$ & $17(35.42 \%)$ & 0.006 & & & \\
\hline $18(36.73 \%)$ & $31(64.58 \%)$ & & & & \\
\hline
\end{tabular}

Data are stratified by the median level of gene expression.

categorical variables were analyzed using the chi square test. DFS and OS survival curves were computed using the Kaplan-Meier method and compared using the log-rank test. Hazard ratios (HR) for disease progression and patient death were estimated using the Cox proportional hazard model. Multivariate analyses were carried out adjusting for patient age, sex, Weiss score, ACC stage, mitotic count and cortisol secretion. Cox models were also used to assess
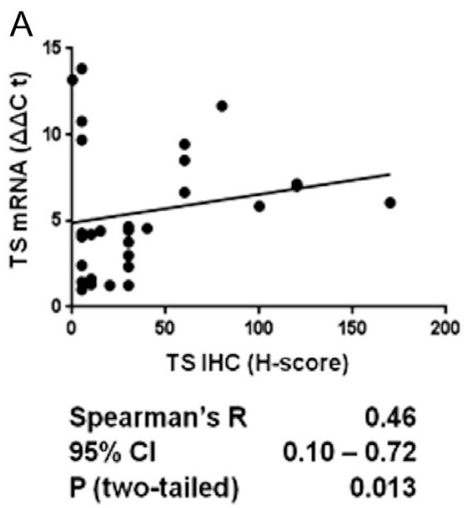
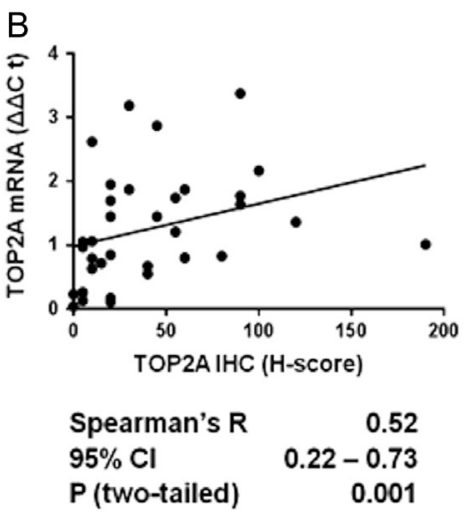
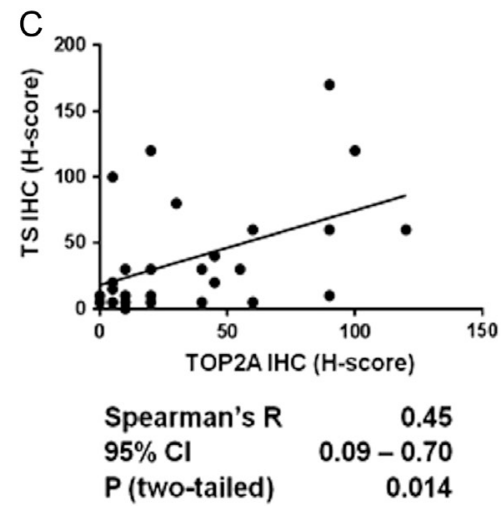

Figure 1

Relationship between mRNA and immohistochemical expression of TOP2A (A) and TS (B). Relationship between TOP2A and TS at protein level (C).

http://erc.endocrinology-journals.org DOI: 10.1530/ERC-17-0095
(C) 2017 Society for Endocrinology Printed in Great Britain
Published by Bioscientifica Ltd. 


\section{Disease Free Survival}

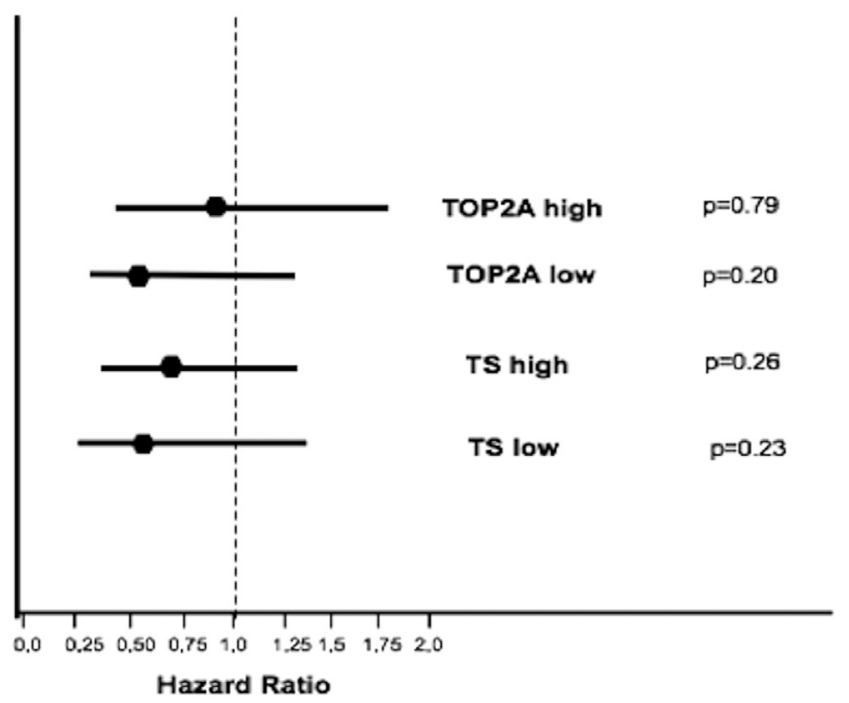

Figure 2

Predictive role of TOP2A and TS expression for the efficacy of adjuvant mitotane. Marker expression is dichotomized at the median value.

the presence of heterogeneity in the effect of marker expression in the different patient subgroups, defined by the covariates, by including in the model the appropriate treatment/covariate interaction term. Statistical analyses were carried out by using the SPSS for windows software (version 17).

\section{Results}

\section{Patients}

Patient characteristics are depicted in Table 1. Both Italian and German cohorts were comparable in terms of age, sex proportion, presence of clinical syndromes and tumor characteristics (data for 92 patients previously published, (Volante et al. 2012). Adjuvant mitotane therapy was administered to 38 patients (38.8\%): 18 Italian and 20 German patients, respectively; the remaining 54 patients (55.1\%) with non-metastatic disease did not receive any postoperative treatment. The median follow-up was 66.3 months in all patients, being 80 months and 62.8 months in the Italian and German cohorts, respectively. Among patients with stage II-III disease, 64 (65.3\%) developed disease recurrence, 29 of the Italian series $(67.4 \%)$ and 35 of the German series (74.5\%). Among them, 30 were treated with chemotherapy; streptozotocyn in 8 patients, EDP (etoposide, doxorubicin, cisplatin) in 22 patients. Both streptozotocyn and EDP were administered in association with mitotane. Overall 51 patients (52.0\%) died of ACC progression: 28 (54.9\%) in the Italian series and 23 (48.9\%) in the German series, respectively.

\section{Relationship between tissue marker expression and patient and tumor characteristics}

As outlined in Table 2, both TOP2A and TS gene expression, dichotomized at the median value, did not show any significant relationship with histopathologic features, except for the significant direct correlation between TS expression levels and Weiss score. TOP2A and TS expressions were significantly directly correlated $(P=0.006)$ and both markers showed a direct relationship with RRM1 ( $P=0.026$ and $P=0.011$ for TOP2A and TS, respectively) but not with ERCC1 $(P=0.54$ and $P=0.61$ for TOP2A and TS, respectively). In the 39 patients in which TOP2A and TS protein expression were assessed by immunohistochemistry, a significant relationship was found between the gene and the relevant protein expression of both enzymes (Spearman R 0.52 and 0.46 for TOP2A and TS, respectively) (Fig. 1).

\section{Relationship between marker expression and disease free and overall survival}

In univariate analysis, TS gene expression was not associated with DFS (Hazard Ratio (HR): 0.96, 95\% Confidence Interval (CI): 0.60-1.53, $P=0.87)$ and OS (0.86, 95\% CI: $0.49-1.50, P=0.60$ ). High TOP2A expression levels were associated with higher risk of disease recurrence but just failing to attain statistical significance (HR: 1.49, 95\% CI: $0.93-2.37, P=0.09)$ and was significantly associated with a higher risk of death (HR: 1.78, 95\% CI: 1.02-3.19, $P<0.05$ ). In multivariate analysis, however, TOP2A expression failed to be a significant independent prognostic parameter either in terms of DFS (HR: 1.18, 95\% CI: $0.71-1.98, P=0.51$ ) or OS (HR: $1.61,95 \% \mathrm{CI}$ : $0.87-2.98, P=0.13)$.

\section{Predictive role of TOP2A and TS expression for the efficacy of adjuvant mitotane}

As previously reported, in this series mitotane-treated patients had a longer median DFS than untreated patients (22.5 months (95\% CI, 1.8-43.1) vs 13.2 months (95\% CI, 6.2-20.2), HR, 0.70 (95\% CI, 0.43-1.16; $P=0.17)$ ) and longer median OS (154 months (95\% CI, 65.1-242.9) vs 53 months (95\% CI, 22.6-83.4), HR 0.63 (95\% CI, $0.34-1.16 ; P=0.14)$ ).

Published by Bioscientifica Ltd. 

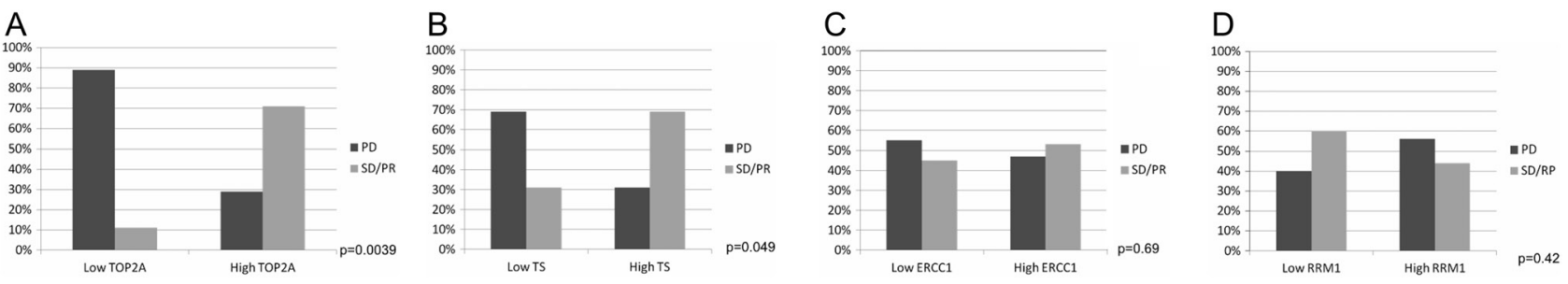

Figure 3

Correlation between markers expression and Clinical Benefit (Partial Response and Disease Stabilization of EDP-M scheme (A) TOP2A, (B) TS, (C) ERCC1, (D) RRM1.

The prognostic effect of mitotane administration was assessed stratifying the patients according to marker expression (dichotomized at the median value). As shown in Fig. 2, no different effect of mitotane treatment was observed in terms of DFS in patients with high vs low TOP2A and TS expression.

\section{Predictive role of marker expression of EDP + mitotane activity}

Among the 26 ACC patients who received first line EDP-M for metastatic disease, four (15\%) achieved a partial response (PR), nine (35\%) stable disease (SD) and 13 (50\%) experienced progressive disease (PD) after 2 or 3 cycles.

The clinical benefit (SD or PR) of EDP-M was directly associated with TOP2A expression: 12 SD/PR out of 17 (71\%) patients in high TOP2A group as opposed to $1 / 9$ $(11 \%)$ in low TOP2A group $(P=0.0039)$ (Fig. 3A). Stratifying patients according to TS expression, a SD/PR was observed in 9/13 (69\%) patients with high gene expression and 4/13 (31\%) patients with low TS expression, respectively $(P=0.049)$ (Fig. 3B). EDP-M administration was associated with longer time to progression (TTP) in patients with high TOP2A as opposed to those with low TOP2A $(P=0.038)$ (Fig. 4A), while no difference in terms of TTP after EDP-M was observed stratifying patients according to TS (data not shown). Fifteen EDP-M treated patients had TOP2A protein expression assessed by immunohistochemistry. TOP2A expression $>30 \%$ of stained cells (the median value) was associated with a greater chance to attain SD/PR (5/9 (55.5\%) vs $1 / 6(16.6 \%), P=0.22)$ and longer TTP (Fig. 4B) than TOP2A $\leq 30 \%$. These differences failed to attain the statistical significance. Among the 12 EDP-M patients, whose tumors had TS expression assessed by immunohistochemistry, TS expression above the median value ( $>30 \%$ of stained cells) was associated with a nonsignificant greater SD/PR rate $(3 / 6(50.0 \%)$ vs $1 / 6$ (16.6\%), $P=0.54$ ) but TTP was similar to that of patients with TS $\leq 30 \%$ (data not shown).
Neither ERCC1 nor RRM1 correlated with a clinical benefit of the therapy: SD/PR in 8/15 (53\%) vs 5/11 (45\%) in high and low ERCC1 expressing tumors $(P=0.69)$ (Fig. 3C); SD/PR in $7 / 16$ (44\%) vs $6 / 10(60 \%)$ in high and low RRM1 expressing tumors, respectively $(P=0.42)$ (Fig. 3D). No difference in term of TTP was observed
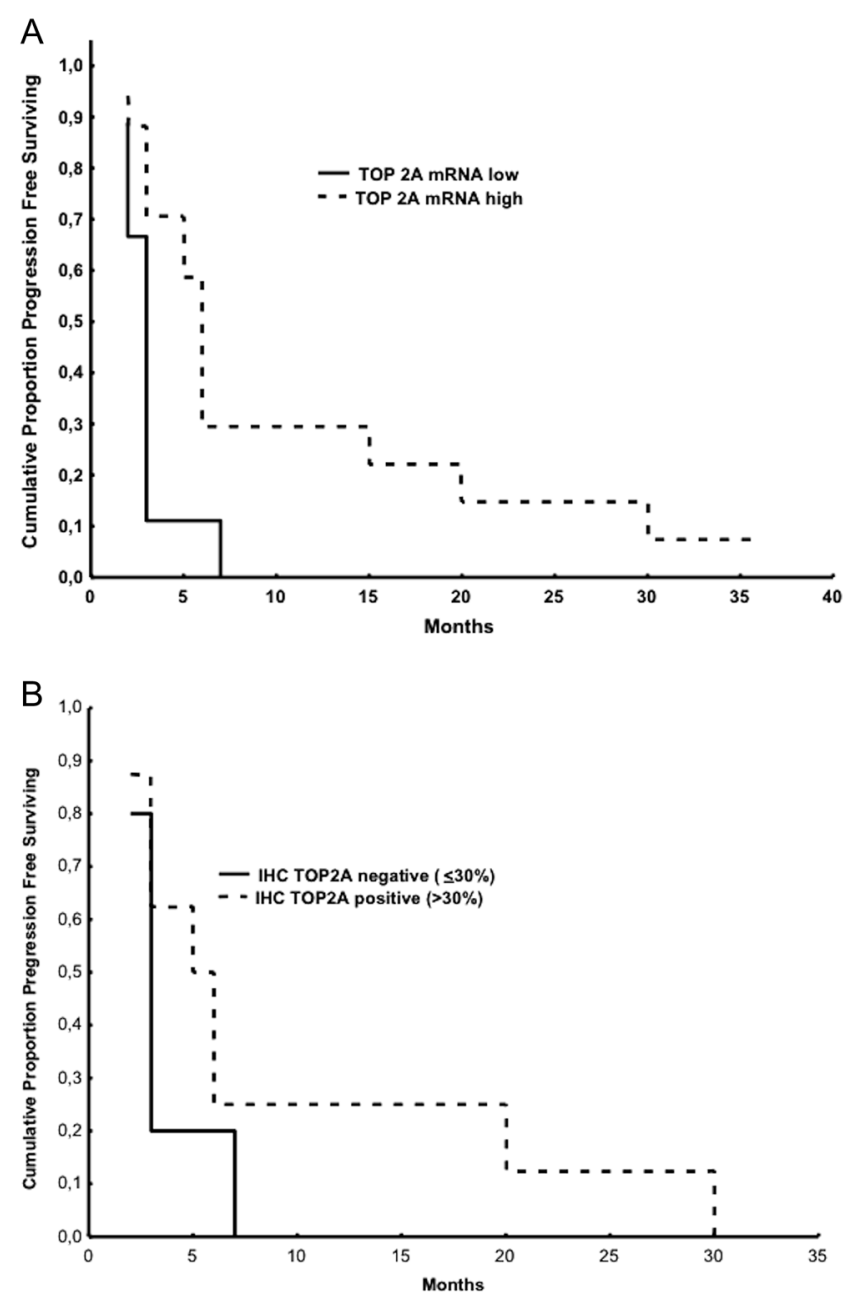

Figure 4

Relationship between TOP2A expression and time to progression (TTP) in patients treated with EDP-M.

Published by Bioscientifica Ltd. 
stratifying patients according to both RRM1 and ERCC1 expression (data not shown).

\section{Discussion}

TOP2A and TS have been shown to play a prognostic role in several cancers, such as colorectal, esophageal, breast, prostate, pancreatic, lung and other cancers (Peters et al. 1994, Lenz et al. 1998, Suda et al. 1999, Suzuki et al. 1999, Edler etal.2000, Harpole etal.2001, Nakopoulou etal.2001, Di Leo \& Isola 2003, Gonen et al. 2003, O'Connor et al. 2006, Shvero et al. 2008, Faggad et al. 2009, Van der Zee et al. 2012, Lee et al. 2013, Yan et al. 2015). At the best of our knowledge, the prognostic role of these two markers has never been explored in ACC.

In this study, we have measured the mRNA expressions of TOP2A and TS in a relatively large series of 98 ACC patients, considering the extreme rarity of this tumor.

The results showed that TS expression is not prognostic in ACC while TOP2A expression in univariate analysis showed a significant correlation with a worse OS although failing to be significantly associated with DFS. In multivariate analysis, after adjusting for prognostic factors including mitotic index, TOP2A expression failed to be a significant independent prognostic parameter. TOP2A is a marker of cell proliferation (Zhang et al. 2014) and the direct correlation of this enzyme expression with the mitotic count (although not significant) could account for its failure to be an independent prognostic parameter.

As mentioned in the introduction, both TOP2A and TS are also well known predictors of chemotherapy efficacy. In our series, high TOP2A expression in primary ACC was significantly associated with responsiveness to EDP-M and increased TTP in metastatic patients.

All these data refer to gene expression of both enzymes. In order to explore whether TOP2A and TS expressed at the protein level provide the same information, the expression of both enzymes by immunohistochemistry was performed in the Italian patients having residual paraffin material available. A significant correlation was found between PCR and immunohistochemistry both for TOP2A and TS expression. In addition, the predictive role of TOP2A of efficacy of EDP-M in advanced ACC patients was confirmed also at immunohistochemistry although without attaining the statistical significance due to the low number of patients considered. Taken together these data suggest that TOP2A and TS protein expression may have the same prognostic and predictive role as mRNA expression levels in ACC.
TOP2A is a notorious predictor of efficacy to anthracycline and topoisomerase inhibitors in several tumors, but not to cisplatin. However, cisplatin is the reference cytotoxic drug in ACC and it is actually unknown whether EDP is more efficacious than cisplatin alone since no randomized studies have been conducted. In our opinion, these data suggest the importance of Adriamycin and etoposide in the EDP scheme, particularly if TOP2A is highly expressed.

Also TS showed a direct relationship with the disease response of EDP-M, although with a lesser magnitude than TOP2A, but not with TTP. Since fluoropyrimidines are not included in the EDP-M regimen, the direct relationship of TS with TOP2A may explain the association of this marker expression with EDP-M activity.

In this small series, both RRM1 and ERCC1 failed to be associated with EDP-M activity, this is an expected finding for RRM1 since this marker was repeatedly found to be predictive of gemcitabine efficacy, but not for ERCC1 that is a predictive factor of cisplatin efficacy in nonsmall cell lung cancer (Ronchi et al. 2009). In ACC patients, tissue ERCC1 assessed by immunohistochemistry was associated with efficacy of cisplatin containing regimens in one study (Baudin et al. 2011) but not confirmed in another series (Sbiera et al. 2015).

In this paper we also explored the potential role of TOP2A and TS for mitotane efficacy in adjuvant setting. Although very recently sterol-O-acyl-transferase 1 (SOAT1) was identified as a key intracellular target of mitotane (Sbiera et al. 2015), the identification of a possible association between mitotane efficacy with a biological parameter in an explorative analysis, would be very useful to understand the mechanisms of sensitivity and resistance of ACC to this drug.

Both TOP2A and TS failed to have a predictive role for adjuvant mitotane efficacy; however the difference in the DFS curves of adjuvant mitotane vs follow-up only in low vs high TS expressing ACC, although not significant, does not exclude the potential role of this marker of mitotane efficacy.

\section{Conclusions}

The predictive role of TOP2A expression with the efficacy of EDP-M is new and of potential clinical relevance. EDP is a toxic regimen and this observation, if confirmed, suggests the potential importance of adding Adriamycin and etoposide to cisplatin in highly expressing TOP2A tumors, while these 2 drugs might be unhelpful in low TOP2A expressing ACC.

Published by Bioscientifica Ltd 


\section{Declaration of interest}

The authors declare that there is no conflict of interest that could be perceived as prejudicing the impartiality of the research reported.

\section{Funding}

This study was supported by grants from AIRC, Milan (no. IG/14820/2013 to M P), Fondazione Camillo Golgi, Brescia, and donations of 'gli Amici di Carlo' in memory of Carlo Ridon, 'gli Amici di Andrea' in memory of Andrea Gadeschi, Pertot Valentino and Stolfa Maria Teresa in memory of Pertot Cristiana, Serena Ambrogini in memory of Guido Cioni.

\section{Author contribution statement}

Elisa Roca, Alfredo Berruti, Massimo Terzolo and Marco Volante contributed to study concept and design and to writing of the report. Silviu Sbiera, Paola Sperone, Barbara Zaggia and Cristina Ronchi were investigators and contributed to acquisition of data and identifications of eligible patients. Ida Rapa and Antonina Germano performed immunohistochemistry analysis, the RNA isolation from paraffin embedded tissues and quantitative real-time PCR. Laura Ferrari and Salvatore Grisanti evaluated, analyzed, and interpreted the data. Martin Fassnacht and Giorgio Vittorio Scagliotti performed a critical revision of the manuscript for important intellectual content. Mauro Papotti contributed to the study concept and design, performed a critical supervision of the study and revised the manuscript. All authors reviewed and edited the report.

\section{References}

Baudin E, Pellegriti G, Bonnay M, Penfornis A, Laplanche A, Vassal G \& Schlumberger M 2001 Impact of monitoring plasma 1,1-dichlorodiphenildichloroethane (o,p'DDD) levels on the treatment of patients with adrenocortical carcinoma. Cancer 92 1385-1392. (doi:10.1002/1097-0142(20010915)92:6<1385::AIDCNCR1461>3.0.CO;2-2)

Baudin E, Leboulleux S, Al Ghuzlan A, Chougnet C, Young J, Deandreis D, Dumont F, Dechamps F, Caramella C, Chanson P, et al. 2011 Therapeutic management of advanced adrenocortical carcinoma: what do we know in 2011? Hormones and Cancer 6 363-371. (doi:10.1007/s12672-011-0094-2)

Bellantone R, Ferrante A, Boscherini M, Lombardi CP, Crucitti P, Crucitti F, Favia G, Borrelli D, Boffi L, Capussotti L, et al. 1997 Role of reoperation in recurrence of adrenal cortical carcinoma: results from 188 cases collected in the Italian National Registry for adrenal cortical carcinoma. Surgery 122 1212-1218. (doi:10.1016/S00396060(97)90229-4)

Berruti A, Fassnacht M, Baudin E, Hammer G, Haak H, Leboulleux S, Skogseid B, Allolio B \& Terzolo M 2010 Adjuvant therapy in patients with adrenocortical carcinoma: a position of an international panel. Journal of Clinical Oncology 28 e401-e402; author reply e403. (doi:10.1200/JCO.2009.27.5958)

Berruti A, Baudin E, Gelderblom H, Haak HR, Porpiglia F, Fassnacht M, Pentheroudakis G \& ESMO Guidelines Working Group 2012 Adrenal cancer: ESMO Clinical Practice Guidelines for diagnosis, treatment and follow-up. Annals of Oncology 23 (Supplement 7) vii131-vii138. (doi:10.1093/annonc/mds231)

Beuschlein F, Weigel J, Saeger W, Kroiss M, Wild V, Daffara F, Libé R, Ardito A, Ghuzlan A, Quinkler M, et al. 2015 Major prognostic role of Ki67 in localized adrenocortical carcinoma after complete resection. Journal of Clinical Endocrinology and Metabolism 100 841-849. (doi:10.1210/jc.2014-3182)
Ceppi P, Longo M, Volante M, Novello S, Cappia S, Bacillo E, Selvaggi G, Saviozzi S, Calogero R, Papotti M, et al. 2008a Excision repair cross complementing-1 and topoisomerase IIalpha gene expression in small-cell lung cancer patients treated with platinum and etoposide: a retrospective study. Journal of Thoracic Oncology 3 583-589. (doi:10.1097/JTO.0b013e3181734f24)

Ceppi P, Volante M, Ferrero A, Righi L, Rapa I, Rosas R, Berruti A, Dogliotti L, Scagliotti GV \& Papotti M 2008b Thymidylate synthase expression in gastroenteropancreatic and pulmonary neuroendocrine tumors. Clinical Cancer Research 14 1059-1064. (doi:10.1158/10780432.CCR-07-1513)

Di Leo A \& Isola J 2003 Topoisomerase II $\alpha$ as a marker predicting the efficacy of anthracyclines in breast cancer: are we at the end of the beginning? Clinical Breast Cancer 4 179-186. (doi:10.1016/s15268209(11)70623-1)

Edler D, Glimelius B, Hallström M, Jakobsen A, Johnston PG, Magnusson I, Ragnhammar P \& Blomgren H 2000 Thymidylate synthase expression: an independent prognostic factor for local recurrence, distant metastasis, disease-free and overall survival in rectal cancer. Clinical Cancer Research 6 1378-1384. (doi:10.1200/ JCO.2002.07.039)

Faggad A, Darb-Esfahani S, Wirtz R, Sinn B, Sehouli J, Könsgen D, Lage H, Weichert W, Noske A, Budczies J, et al. 2009 Topoisomerase II $\alpha$ mRNA and protein expression in ovarian carcinoma: correlation with clinicopathological factors and prognosis. Modern Pathology 22 579-588. (doi:10.1038/modpathol.2009.14)

Fassnacht M, Johanssen S, Quinkler M, Bucsky P, Willenberg HS, Beuschlein F, Terzolo M, Mueller HH, Hahner S, Allolio B, et al. 2009 Limited prognostic value of the 2004 International Union Against Cancer staging classification for adrenocortical carcinoma: proposal for a revised TNM classification. Cancer 115 243-250. (doi:10.1002/ cncr.24030)

Fassnacht M, Libé R \& Kroiss M 2011 Adrenocortical carcinoma: a clinician's update. Nature Reviews Endocrinology 7 323-335. (doi:10.1038/nrendo.2010.235)

Fassnacht M, Terzolo M, Allolio B, Baudin E, Haak H, Berruti A, Welin S, Schade-Brittinger C, Lacroix A, Jarzab B, et al. 2012 Combination chemotherapy in advanced adrenocortical carcinoma. New England Journal of Medicine 366 2189-2197. (doi:10.1056/NEJMoa1200966)

Formentini A, Henne-Bruns D \& Kornmann M 2004 Thymidylate synthase expression and prognosis of patients with gastrointestinal cancers receiving adjuvant chemotherapy: a review. Langenbeck's Archives of Surgery 389 405-413. (doi:10.1007/s00423-004-0510-y)

Gonen M, Hummer A, Zervoudakis A, Sullivan D, Fong Y, Banerjee D, Klimstra D, Cordon-Cardo C, Bertino J \& Kemeny N 2003 Thymidylate synthase expression in hepatic tumors is a predictor of survival and progression in patients with resectable metastatic colorectal cancer. Journal of Clinical Oncology 21 406-412. (doi:10.1200/JCO.2003.06.060)

Hahner S \& Fassnacht M 2005 Mitotane for adrenocortical carcinoma treatment. Current Opinion in Investigational Drugs 6 386-394.

Harpole DH Jr, Moore MB, Herndon JE 2nd, Aloia T, D'Amico TA, Sporn T, Parr A, Linoila I \& Allegra C 2001 The prognostic value of molecular marker analysis in patients treated with trimodality therapy for esophageal cancer. Clinical Cancer Research 7 562-569.

Icard P, Goudet P, Charpenay C, Andreassian B, Carnaille B, Chapuis Y, Cougard P, Henry JF \& Proye C 2001 Adrenocortical carcinomas: surgical trends and results of a 253-patient series from the French Association of Endocrine Surgeons study group. World Journal of Surgery 25 891-897. (doi:10.1007/s00268-001-0047-y)

Kellner U, Sehested M, Jensen PB, Gieseler F \& Rudolph P 2002 Culprit and victim - DNA topoisomerase II. Lancet Oncology 3 235-243. (doi:10.1016/S1470-2045(02)00715-5) http://erc.endocrinology-journals.org

DOI: 10.1530/ERC-17-0095
(C) 2017 Society for Endocrinology Printed in Great Britain
Published by Bioscientifica Ltd 
Lee SW, Chen TJ, Lin LC, Li CF, Chen LT, Hsing CH, Hsu HP, Tsai CJ, Huang HY \& Shiue YL 2013 Overexpression of thymidylate synthetase confers an independent prognostic indicator in nasopharyngeal carcinoma. Experimental and Molecular Pathology 95 83-90. (doi:10.1016/j.yexmp.2013.05.006)

Lenz HJ, Hayashi K, Salonga D, Danenberg KD, Danenberg PV, Metzger R, Banerjee D, Bertino JR, Groshen S, Leichman LP, et al. 1998 p53 point mutations and thymidylate synthase messenger RNA levels in disseminated colorectal cancer: an analysis of response and survival. Clinical Cancer Research 4 1243-1250.

Lv YT, Du PJ, Wang QY, Tan Y, Sun ZB, Su ZL \& Kang CM. 2013 A novel approach to cloning and expression of human thymidylate synthase. Asian Pacific Journal of Cancer Prevention 14 7523-7527. (doi:10.7314/ APJCP.2013.14.12.7523)

Nakopoulou L, Zervas A, Lazaris AC, Constantinides C, Stravodimos C, Davaris P \& Dimopoulos C 2001 Predictive value of topoisomerase Ii $\alpha$ immunostaining in urothelial bladder carcinoma. Journal of Clinical Pathology 54 309-313. (doi:10.1136/jcp.54.4.309)

O'Connor JK, Hazard LJ, Avent JM, Lee RJ, Fischbach J \& Gaffney DK 2006 Topoisomerase II alpha expression correlates with diminished disease free survival in invasive breast cancer. International Journal of Radiation Oncology, Biology, Physics 65 1411-1415. (doi:10.1016/j. ijrobp.2006.02.045)

Peters GJ, van der Wilt CL, van Groeningen CJ, Smid K, Meijer S \& Pinedo HM 1994 Thymidylate synthase inhibition after administration of fluorouracil with or without leucovorin in colon cancer patients: implications for treatment with fluorouracil. Journal of Clinical Oncology 12 2035-2042. (doi:10.1200/JCO.1994.12.10.2035)

Ronchi CL, Sbiera S, Kraus L, Wortmann S, Johanssen S, Adam P, Willenberg HS, Hahner S, Allolio B \& Fassnacht M 2009 Expression of excision repair cross complementing group 1 and prognosis in adrenocortical carcinoma patients treated with platinum-based chemotherapy. Endocrine-Related Cancer 16 907-918. (doi:10.1677/ ERC-08-0224)

Sbiera S, Leich E, Liebisch G, Sbiera I, Schirbel A, Wiemer L, Matysik S, Eckhardt C, Gardill F, Gehl A, et al. 2015 Mitotane inhibits sterol-Oacyl transferase 1 triggering lipid-mediated endoplasmic reticulum stress and apoptosis in adrenocortical carcinoma cells. Endocrinology 156 3895-3908. (doi:10.1210/en.2015-1367)

Shvero J, Koren R, Shvili I, Yaniv E, Sadov R \& Hadar T 2008 Expression of human DNA topoisomerase ii- $\alpha$ in squamous cell carcinoma of the larynx and its correlation with clinicopathologic variables. American Journal of Clinical Pathology 130 934-939. (doi:10.1309/ AJCPROG61USKCBEI)

Suda Y, Kuwashima Y, Tanaka Y, Uchida K \& Akazawa S 1999 Immunohistochemical detection of thymidylate synthase in advanced gastric cancer: a prognostic indicator in patients undergoing gastrectomy followed by adjuvant chemotherapy with 5-fluoropyrimidines. Anticancer Research 19 805-810.
Suzuki M, Tsukagoshi S, Saga Y, Ohwada M \& Sato I 1999 Enhanced expression of thymidylate synthase may be of prognostic importance in advanced cervical cancer. Oncology 57 50-54. (doi:10.1159/ 000012000)

Terzolo M, Angeli A, Fassnacht M, Daffara F, Tauchmanova L, Conton PA, Rossetto R, Buci L, Sperone P, Grossrubatscher E, et al. 2007 Adjuvant mitotane treatment for adrenocortical carcinoma. New England Journal of Medicine 356 2372-2380. (doi:10.1056/ NEJMoa063360)

Terzolo M, Baudin AE, Ardito A, Kroiss M, Leboulleux S, Daffara F, Perotti P, Feelders RA, deVries JH, Zaggia B, et al. 2013 Mitotane levels predict the outcome of patients with adrenocortical carcinoma treated adjuvantly following radical resection. European Journal of Endocrinology 169 263-270. (doi:10.1530/EJE-13-0242)

Terzolo M, Daffara F, Ardito A, Zaggia B, Basile V, Ferrari L \& Berruti A 2014 Management of adrenal cancer: a 2013 update. Journal of Endocrinological Investigation 37 207-217. (doi:10.1007/s40618-0130049-2)

Van der Zee JA, van Eijck CH, Hop WC, van Dekken H, Dicheva BM, Seynhaeve AL, Koning GA, Eggermont AM \& Ten Hagen TL 2012 Expression and prognostic significance of thymidylate synthase (TS) in pancreatic head and periampullary cancer. European Journal of Surgical Oncology 38 1058-1064. (doi:10.1016/j.ejso.2012.04.013)

Volante M, Berruti A \& Papotti M 2008 Pathological and molecular features of adrenocortical carcinoma: an update. Journal of Clinical Pathology 61 787-793. (doi:10.1136/jcp.2007.050625)

Volante M, Terzolo M, Fassnacht M, Rapa I, Germano A, Sbiera S, Daffara F, Sperone P, Scagliotti G, Allolio B, et al. 2012 Ribonucleotide reductase large subunit (RRM1) gene expression may predict efficacy of adjuvant mitotane in adrenocortical cancer. Clinical Cancer Research 18 3452-3461. (doi:10.1158/1078-0432.CCR11-2692)

Wang J, Xu B, Yuan P, Zhang P, Li Q, Ma F \& Fan Y 2012 TOP2A amplification in breast cancer is a predictive marker of anthracycline-based neoadjuvant chemotherapy efficacy. Breast Cancer Research and Treatment 135 531-537. (doi:10.1007/s10549012-2167-5)

Weiss LM, Medeiros LJ \& Vickery Al Jr 1989 Pathologic features of prognostic significance in adrenocortical carcinoma. American Journal of Surgical Pathology 13 202-202. (doi:10.1097/00000478-19890300000004)

Yan H, Guo X, Wang H \& Zhu T 2015 Predictive value of thymidylate synthase for the prognosis and survival of lung adenocarcinoma patients. Oncology Letters 9 252-256. (doi:10.3892/ol.2014.2658)

Zhang H, Li J, Zhang Y, Sun M, Zhao P, Zhang G, Jin C, Sun L, He M, Wang B, et al. 2014 ERCC1 mRNA expression is associated with the clinical outcome of non-small cell lung cancer treated with platinum-based chemotherapy. Genetics and Molecular Research 13 10215-10222. (doi:10.4238/2014.December.4.16)

Received in final form 22 March 2017

Accepted 21 April 2017

Accepted Preprint published online 21 April 2017
(C) 2017 Society for Endocrinology Printed in Great Britain
Published by Bioscientifica Ltd. 